\title{
Tratamiento "híbrido" del aneurisma tóraco-abdominal: revascularización visceral extraanatómica e inserción de endoprótesis
}

\author{
Renato Mertens $\mathrm{M}^{1}$, Francisco Valdés $\mathrm{E}^{\mathbf{1}}$, Albrecht Krämer Sch${ }^{1}$, \\ Leopoldo Mariné $M^{1}$, Michel Bergoeing $R^{1}$, \\ Rodrigo Sagües $C^{1}$, Alvaro H uete $\mathbf{G}^{2}$, Jeannette Vergara $G^{a}$, \\ Magaly Valdebenito $\mathrm{G}$ a.
}

\section{Extra anatomical revascularization and endovascular stent-grafting for thoracoabdominal aneurysm repair. Report of four cases}

Surgical treatment of thoracoabdominal aneurysms is a big technical challenge with a high rate of complications and mortality. It requires a large exposure and transient interruption of vital organ perfusion during its repair. Endovascular repair is a less invasive alternative available over the last decade. We report four male patients aged 44 to 76 years, with thoracic aortic aneurysms and involvement of visceral aorta, treated with a two stage procedure. During the first stage, a retrograde revascularization of the superior mesenteric and renal arteries from the infrarenal aorta was done, associated in two cases to a concomitant repair of an infrarenal aortic aneurysm. In the second stage, an endovascular graft was placed through the femoral artery, from the segment proximal to the aneurysm to the infrarenal aorta, above the origin of the visceral artery reconstructions, excluding the aneurysm from circulation. In one patient, both stages were concomitant and in three the second stage was delayed. One patient presented a postoperative bleeding that required reintervention without adverse consequences. No patient died, presented paraplegia or deterioration of renal function. After follow up of 6 to 20 months, there is no evidence of aneurysm growth or complications derived from the procedure (Rev Méd Chile 2007; 135: 153-9).

(Key words: Aortic aneurysm; Endovascular stent-grafting; Stents)

Recibido el 9 de junio, 2006. Aceptado el 2 de agosto, 2006.

${ }^{1}$ Cirugía Vascular y Endovascular, Departamentos de Enfermedades Cardiovasculares y División de Cirugía. ${ }^{2}$ Departamento de Radiología, Pontificia Universidad Católica de Chile. aEnfermera Universitaria

Correspondencia a: Dr. Renato Mertens M. Apoquindo 3990, oficina 601, Santiago. Fono: (56 2) 686 3268. Fax:

(56 2) 632 6812. E mail: rmertens@med.puc.cl 
L a a historia natural del aneurisma de la aorta tóraco-abdominal es hacia la ruptura y muerte por hemorragia, dependiendo del diámetro de la lesión ${ }^{1,2}$.

El tratamiento quirúrgico convencional de estas lesiones implica un extenso abordaje quirúrgico y detener el flujo sanguíneo hacia el sector visceral abdominal por un tiempo variable. Esto conlleva una alta morbimortalidad, donde destaca una alta incidencia de paraplejia definitiva por isquemia medular ${ }^{3-5}$.

En los últimos años, el desarrollo de técnicas endovasculares mínimamente invasivas para el tratamiento de las enfermedades de la aorta ha abierto una opción menos agresiva para el manejo de estos pacientes ${ }^{6}$.

Las endoprótesis comercialmente disponibles en la actualidad son dispositivos tubulares relativamente simples, su aplicación aislada en esta situación llevaría a cubrir el origen de los vasos viscerales y renales provocando isquemia. Con el fin de hacer técnicamente posible el procedimiento endovascular en este tipo de pacientes, hemos realizado primero revascularización quirúrgica extraanatómica de estos vasos, previo a la inserción de la endoprótesis.

A continuación reportamos nuestra experiencia acumulada en la aplicación de esta técnica en esta grave condición.

\section{MATERIAL Y MÉTODO}

Se consideraron candidatos a este procedimiento pacientes con lesiones aneurismáticas de aorta descendente con compromiso de la aorta visceral.

Los pacientes fueron evaluados mediante tomografía axial computada helicoidal multicorte con contraste en fase arterial, obteniendo reconstrucciones axiales cada $3,75 \mathrm{~mm}$ o menos, con la posibilidad de obtener reconstrucciones coronales o sagitales (Figura 1A). Los criterios para ser incluidos fueron:

1. Factibilidad de acceso a través de las arterias femorales o ilíacas para introducir un dispositivo de alto diámetro, pudiendo llegar a $26 \mathrm{~F}(8,6 \mathrm{~mm})$. 2. Presencia de un segmento no comprometido de aorta para la fijación proximal de la endoprótesis de al menos $15 \mathrm{~mm}$, inmediatamente distal al origen de la arteria carótida común izquierda en el arco aórtico, considerando la necesidad de ocluir el origen de la arteria subclavia izquierda en caso de requerirlo. 3. Un segmento de aorta abdominal no comprometida o prótesis aórtica de dacrón infrarrenal distal a la lesión, en caso de reconstrucción previa de dicho segmento, de al menos $15 \mathrm{~mm}$ para la fijación distal de la endoprótesis. 4 . La posibilidad de adherir a un protocolo estricto de seguimiento a largo plazo.

La evaluación preoperatoria incluyó los parámetros básicos necesarios para un procedimiento de esta envergadura.

Todos los procedimientos fueron realizados en pabellón quirúrgico, bajo anestesia general y monitorización invasiva.

En la primera fase del procedimiento se realizó una laparotomía media tradicional, a través de la cual se realizó revascularización extraanatómica retrógrada de la arteria mesentérica superior y de las arterias renales con prótesis de dacrón, dejando el origen del puente en un lugar alejado y distal a la zona donde se programa el apoyo del extremo distal de la endoprótesis. Una vez revascularizadas, las arterias nativas fueron interrumpidas en su origen para evitar reflujo hacia el saco del aneurisma con eventual presurización (Figura 1B). El tronco celíaco no fue revascularizado y fue cubierto con la endoprótesis en todos los casos. En caso de reconstrucción concomitante de la aorta infrarrenal por aneurisma en esa zona (Figura 2), la arteria mesentérica inferior fue reimplantada en la prótesis, asegurando, de esta forma, permeabilidad de al menos 2 de los 3 vasos viscerales.

La instalación de la endoprótesis tóraco-abdominal fue planificada en un segundo tiempo según la envergadura del procedimiento original y endovascular.

Dicha intervención fue realizada bajo anestesia general, con la monitorización habitual y utilizando un catéter espinal para drenar y controlar la presión de líquido cefalorraquídeo como profilaxis de paraplejia, de la forma que se ha descrito en la literatura ${ }^{7} \mathrm{y}$ hemos aplicado en el pasado ${ }^{8}$.

La prótesis fue insertada a través de denudación y arteriotomía de la arteria femoral común, seguida de su reparación quirúrgica ya que el gran calibre del dispositivo impide su uso percutáneo. El procedimiento fue realizado bajo heparinización sistémica. Se utilizó radioscopia y angiografía 


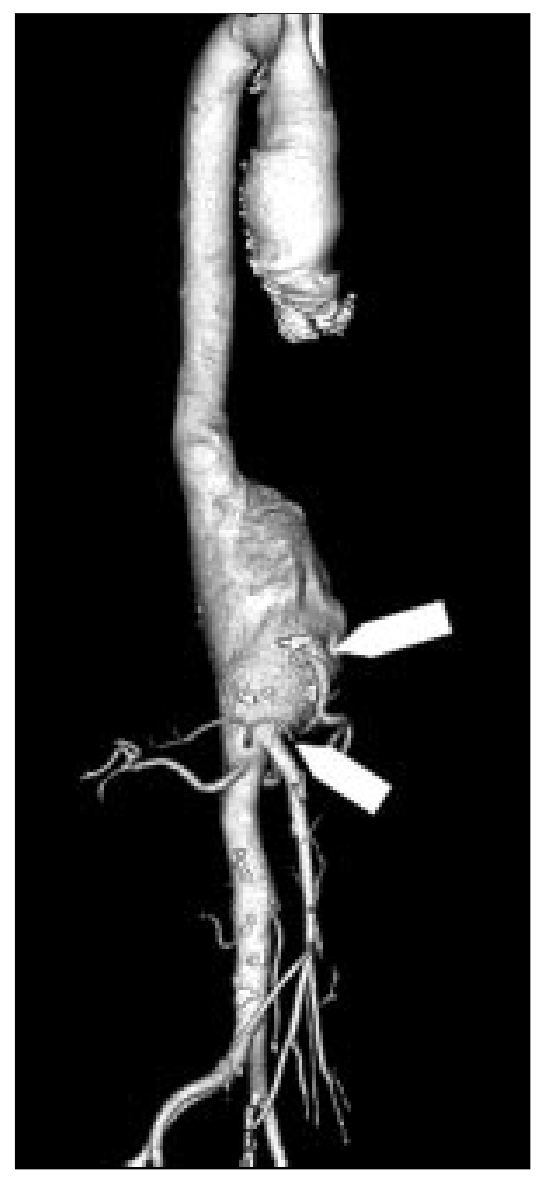

Figura 1A. Reconstrucción tridimensional de tomografía computada en fase arterial en orientación oblicua anterior derecha, demuestra la presencia de un gran aneurisma toracoabdominal con compromiso de las arterias viscerales (flechas).

por sustracción digital para orientar y desplegar la endoprótesis en el lugar apropiado. Para la generación de las imágenes intraoperatorias se utilizó un angiógrafo digital OEC serie 9.800 (General Electric $^{\circledR}$, EE.UU.). La mortalidad operatoria fue definida como intrahospitalaria 0 antes de 30 días. Endofuga fue definida como persistencia de flujo al interior del saco aneurismático, detectada mediante tomografía axial computada. Utilizamos la nomenclatura habitual reconocida para este tipo de complicaciones: Tipo I son filtraciones a través del sitio de anclaje de la endoprótesis en la aorta,

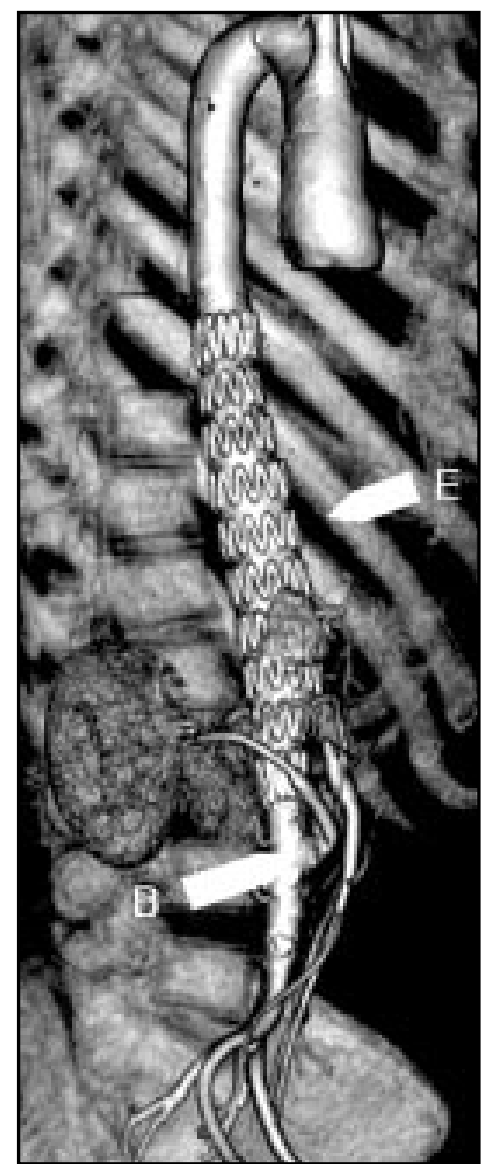

Figura 1B. Reconstrucción del mismo paciente en el posoperatorio. La letra $\mathrm{E}$ muestra la endoprótesis in situ y la letra $\mathrm{B}$ el origen de los puentes retrógrados a la arteria mesentérica superior y ambas arterias renales.

ya sea proximal o distalmente, las tipo II son dependientes de ramas que se originan del segmento de aorta tratado, tipo III se producen por falla estructural de la endoprótesis.

Antes de los 30 días del posoperatorio se realizó una tomografía axial computada helicoidal con contraste en fase arterial (Figuras 1B y 2) para evaluar la localización de la endoprótesis, la presencia de endofugas y la permeabilidad de las reconstrucciones viscerales y renales. Si la tomografía resultaba satisfactoria se programaba un control a los 6 y 12 meses, luego anualmente. 


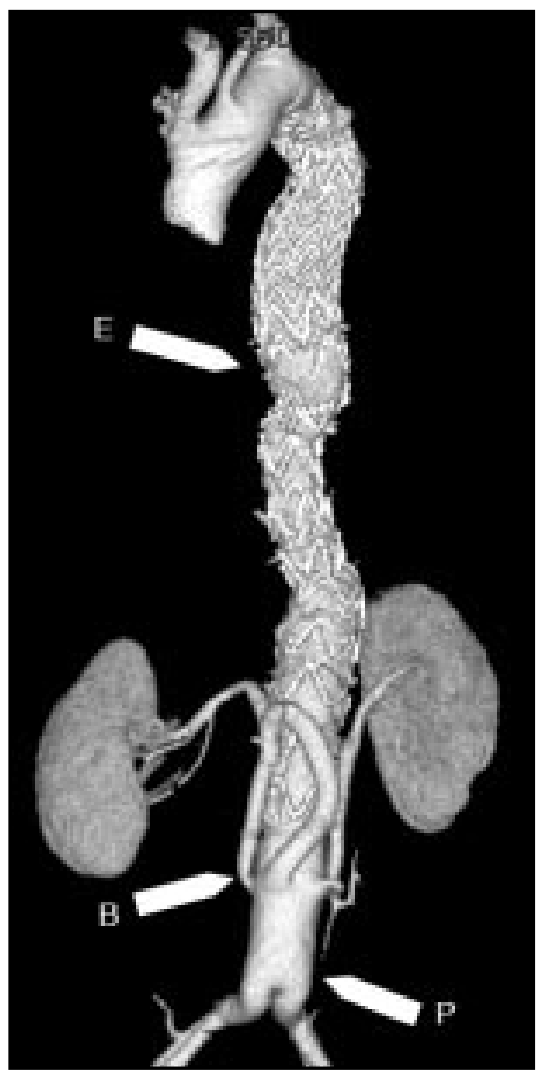

Figura 2. Reconstrucción tridimensional de tomografía computada en fase arterial, muestra una extensa reconstrucción de toda la aorta torácica descendente, visceral e infrarrenal, mediante endoprótesis (E) en los segmentos proximales, prótesis de dacrón bifurcada tradicional en la aorta infrarrenal $(\mathrm{P})$ y puentes viscerales retrógrados (B).

\section{Resultados}

En el periodo comprendido entre diciembre 2004 y diciembre 2005 se trataron 4 pacientes con esta técnica. Todos de sexo masculino, con un promedio de edad de 62,8 años (rango 44-76). Dos pacientes eran hipertensos en tratamiento y uno cardiópata con insuficiencia valvular aórtica y fibrilación auricular crónica.

La etiología del aneurisma toracoabdominal era degenerativo (ateroesclerótico) en 2 pacientes, un paciente era portador de un síndrome de Marfán y el restante disección aórtica crónica con extensa dilatación aneurismática secundaria. El diámetro promedio de los aneurismas fue de 7,2 cm (rango: 5,5-8,3).

Dos pacientes tenían antecedentes de reparación previa de un aneurisma de aorta torácica descendente proximal, en un caso mediante endoprótesis, presentando dilatación gradual del segmento distal y visceral con formación de una endofuga tipo I y en el otro caso (síndrome de Marfán), reparación mixta abierta y endovascular, presentando luego dilatación de aorta torácica distal y abdominal visceral e infrarrenal. En la primera fase de la intervención, a dos pacientes se les realizó reparación simultánea de un aneurisma de aorta abdominal inframenal de diámetros 4,6 y $5 \mathrm{~cm}$, mediante bypass aorto bi-ilíaco con prótesis de dacrón. A estos dos pacientes se les asoció, además de la revascularización renal bilateral y de arteria mesentérica superior, el reimplante de la arteria mesentérica inferior en la prótesis aórtica de dacrón.

Todos los puentes viscerales o renales fueron construidos con prótesis tubulares de dacrón. A un paciente se le realizó el procedimiento endovascular en forma concomitante por considerarse técnicamente factible y simple. En los restantes fue diferido por 9, 48 y 138 días, éste último paciente lo postergó por razones extramédicas. Todos los pacientes inicialmente planificados para corrección en dos etapas fueron completados con el tratamiento endovascular.

Se utilizó el dispositivo Talent (Medtronic ${ }^{\circledR}$, EE.UU.) en 2 pacientes y el dispositivo TX2 (Cook ${ }^{\circledR}$, EE.UU.) en los dos restantes. En dos pacientes se cubrió completamente la aorta torácica descendente. En un paciente fue necesario cubrir el origen de la arteria subclavia izquierda, en el arco aórtico, para estabilizar proximalmente el dispositivo en la aorta, evolucionando libre de claudicación intermitente de la extremidad superior o de síntomas neurológicos por robo vértebro-basilar. Dos pacientes no requirieron de transfusiones y uno sólo de 2 unidades de glóbulos rojos. Un paciente presenta hemoperitoneo posoperatorio luego de la revascularización visceral y reparación simultánea de un aneurisma de aorta abdominal infrarrenal que requirió reintervención, evolucionando sin incidentes y requiriendo 6 unidades de glóbulos rojos o sangre. 
Un paciente presentó alza transitoria de la creatinina plasmática de 1,5 a $2,2 \mathrm{mg} \%$ por $48 \mathrm{~h}$ luego de la revascularización renal, regresando rápidamente a su basal. El resto de los pacientes no modificaron su función renal. No hubo mortalidad operatoria ni déficit neurológico posoperatorio. Todos los pacientes han sido seguidos clínica y radiológicamente por un promedio de 11,5 meses (rango: 6 a 20 meses). Todos los pacientes se encuentran vivos y reintegrados a su vida normal. Ningún aneurisma ha aumentado su diámetro o presentado complicaciones derivadas de la endoprótesis. Dos pacientes presentaron endofuga tipo II pequeña, a nivel del origen del tronco celíaco. Las 14 reconstrucciones viscerales o renales se encuentran permeables, sin evidencias de estenosis.

\section{Discusión}

El tratamiento quirúrgico tradicional del aneurisma tóraco-abdominal tiene una alta morbimortalidad.

La mortalidad de la cirugía electiva en series no seleccionadas puede llegar a ser tan alta como $20,3 \%$, con una morbilidad que supera el $60 \%{ }^{3}$. Las principales complicaciones son la hemorragia, infarto miocárdico, insuficiencia respiratoria, insuficiencia renal aguda y paraplejia por isquemia medular, siendo esta última reportada hasta en $19 \% 3,4$. Esta alta tasa de complicaciones aumenta en relación inversa a la experiencia del equipo tratante $^{9}$. En los últimos años el desarrollo de las técnicas endovasculares para el tratamiento de aneurismas y otras lesiones crónicas de la aorta torácica, han permitido disminuir en forma considerable las cifras de morbimortalidad asociadas al manejo de estas lesiones, llegando a mortalidades inferiores a $6 \%$ y en múltiples series menores a $2 \% 10-17$, lo que ha ocurrido también en nuestra experiencia ${ }^{8,18}$.

Estos resultados han motivado el desarrollo de técnicas que permitan realizar la colocación de una endoprótesis extendiéndola hacia el sector visceral, desde donde nacen importantes ramas, las que no son posibles de ocluir sin producir isquemia importante de esos órganos ${ }^{19}$. Los resultados de este procedimiento han sido promisorios, aunque no ha estado ausente la controversia ${ }^{20}$. El procedimiento inicial de revascularización extraanatómica de los vasos viscerales y renales es una intervención mayor, pero relativamente rutinaria. Por otro lado, una laparotomía no se compara en cuanto a dolor posoperatorio y morbilidad cardiopulmonar con la apertura simultánea del tórax, diafragma y abdomen que requiere el procedimiento tradicional. La revascularización visceral y renal no tiene riesgo conocido de paraplejia en sí mismo y la isquemia de los órganos es breve y controlada, como lo demuestra el grado de estabilidad de la función renal en el pre y posoperatorio en la presente serie. La durabilidad de estas reconstrucciones, aunque desconocida en esta situación especial, ha demostrado ser satisfactoria para el tratamiento de la enfermedad oclusiva, tanto en revascularización renal como mesentéri$\mathrm{ca}^{21-23}$.

Por otra parte, el tratamiento endovascular del aneurisma de aorta torácica evita el clampeo aórtico, periodos de hipotensión global o del hemicuerpo inferior y no ocluye temporal o definitivamente importantes vías colaterales de la circulación de la médula espinal, como las arterias hipogástricas, disminuyendo así el riesgo de isquemia de ésta y en consecuencia de paraplejia. Esta complicación ha sido reportada sólo ocasionalmente en el tratamiento endovascular electivo de aneurismas de la aorta descendente, pero con mucha menor frecuencia que en la cirugía abierta ${ }^{10,11}$. Incluso en algunas series no se reportan casos de paraplejia ${ }^{8,13-15} 0$ incidencias inferiores a $2,5 \% 16,17$. En la presente serie, aunque pequeña, no tuvimos que lamentar esta devastadora complicación. La alternativa de registrar la presión y drenar líquido céfalo-raquídeo en caso de elevación por sobre $10 \mathrm{~cm} \mathrm{H}_{2} \mathrm{O}$ es una maniobra ampliamente aceptada para la prevención de paraplejia en la cirugía abierta del aneurisma tóraco-abdominal ${ }^{7}$, extrapolando dicha experiencia hemos aplicado este procedimiento a nuestros pacientes tratados por lesiones torácicas con esta técnica.

Aunque el resultado a mediano plazo ha sido excelente, la evolución a largo plazo es desconocida. La eventual falla del dispositivo, como la fractura de los stents metálicos o desgarro de la tela impermeable 24 , puede ser detectada en el estudio seriado con imágenes de modo que pueda ser tratado en forma electiva, en general mediante una nueva intervención endovascular. 
Por otro lado, la cirugía abierta no está exenta de complicaciones a largo plazo, el seguimiento riguroso de pacientes sometidos a cirugía abierta tradicional de un aneurisma tóraco-abdominal, ha demostrado la aparición de lesiones en la aorta remanente en $8 \%$ y de complicaciones protésicas en $3 \%$ a 26 meses de seguimiento, requiriendo éstas de reintervención ${ }^{25}$.

No hay duda que con el permanente desarrollo tecnológico es muy posible que este procedimiento «íbrido»quede obsoleto o que su uso sea de excepción. Se encuentran en etapa de evaluación endoprótesis con ramas o fenestraciones

\section{REFERENCIAS}

1. Davies R, Goldstein L, Coady M, Tittle S, Rizzo J, KoPF G ET AL. Yearly rupture or dissection rates for thoracic aortic aneurysms: simple prediction based on size. Ann Thorac Surg 2002; 73: 17-27.

2. Gripe R, Ergin M, Galla J, Lansman S, McCulough J, NGUYen K et al. Natural history of descending thoracic and thoracoabdominal aneurysms. Ann Thorac Surg 1999; 67: 1927-30.

3. Derrow A, Seeger J, Dame D, Carter R, Ozaki C, FLYNN T ET AL. The outcome in the United States after thoracoabdominal aortic aneurysm repair, renal artery bypass, and mesenteric revascularization. J Vasc Surg 2001; 34: 54-61.

4. Crawford E, Crawford J, Safi H. Thoracoabdominal Aortic Aneurysms: Preoperative and Intraoperative Factors Determining Immediate and Long-Term Results of Operations in 605 Patients. J Vasc Surg 1986; 3: 389-404.

5. Cox G, O’Hara P, Hertzer N, Piedmonte M, KrajewsKI L, BeVEN E. Thoracoabdominal Aneurysm Repair: A Representative Experience. J Vasc Surg 1992; 15: 780-8.

6. Mertens R, Valdés F, Krämer A. Tratamiento Endovascular del Aneurisma de Aorta. Revista Chilena de Cirugía 2004; 56: 3-11.

7. Cosem J, Lemaire S, Koksoy C, Schmittung Z, CuRLing P. Cerebrospinal fluid drainage reduces paraplegia after thoracoabdominal aortic aneurysm repair: results of a randomized clinical trial. J Vasc Surg 2002; 35: 631-9. especialmente diseñadas para acomodar las ramas de la aorta visceral y, de esta forma, excluir el aneurisma sin requerir de cirugía abierta para revascularizar estos vasos previamente ${ }^{26,27}$. Estos dispositivos son de alto costo, se encuentran aún en fase experimental y aunque se han reportado resultados iniciales promisorios, no están exentos de problemas ${ }^{28}$.

Nuestros resultados inmediatos y a mediano plazo con el uso de este procedimiento nos han permitido considerar a esta técnica como una excelente opción en el tratamiento de esta grave patología en pacientes seleccionados.

8. Mertens R, Valdés F, Krämer A, Mariné L, Irarrázaval M, Morán S et aL. Tratamiento Endovascular del Aneurisma de Aorta Torácica Descendente. Rev Méd Chile 2003; 131: 390-6.

9. Cowan J Jr, Dimick J, Henke P, Huber T, Stanley J, UрсниRCh G. Surgical treatment of intact thoracoabdominal aortic aneurysms in the United States: hospital and surgeon volume-related outcomes. J Vasc Surg 2003; 37: 1169-74.

10. Temudom T, D'Ayala M, Marín M. Endovascular grafts in the treatment of thoracic aortic aneurysms and pseudoaneurysm. Ann Vasc Surg 2000; 14: 230-8.

11. Greenberg R, Resch T, Nyman U. Endovascular repair of descending thoracic aortic aneurysms: an early experience with intermediate term follow up. J Vasc Surg 2000; 31: 147-56.

12. Ehrlich M, Grabenwoeger M, Cartes-Zumeizu F. Endovascular stent graft repair for aneurysms on the descending thoracic aorta. Ann Thorac Surg 1998; 66: 19-24.

13. Thompson C, Gaxotte V, Rodríguez J, Ramaiah V, VRANIC M, Ravi R ET AL, Endoluminal stent grafting of the thoracic aorta: initial experience with the Gore Excluder. J Vasc Surg 2002; 35: 1163-70.

14. Cambria R, Brewster D, Lauterbach S, Kaufman J, Geuler S, FAN C ET AL. Evolving expenience with thoracic aortic stent graft repair. J Vasc Surg 2002; 35: 1129-36.

15. Hejjmen R, Deblier I, Moll F, Dossche K, van den Berg J, Overtoom T et aL. Endovascular stentgrafting for descending thoracic aortic aneurysms. Eur J Cardiothorac Surg 2002; 21: 5-9. 
16. Ellozy S, Carroccio A, Minor M, Jacobs T, Chae K, Cha A ET AL. Challenges of endovascular tube graft repair of thoracic aortic aneurysm: midterm follow-up and lessons leamed. J Vasc Surg 2003; 38: 676-83.

17. Ishida M, Kato N, Hirano T, Cheng S, Shimono T, TAKEDA K. Endovascular Stent-Graft Treatment for Thoracic Aortic Aneurysms: Short to Midterm Results. J Vasc Interv Radiol 2004; 15: 361-7.

18. Mertens R, Valdés F, Krämer A, Bergoeing M, ZalaquetT R, Baeza C et al. Tratamiento endovascular del trauma de aorta torácica descendente. Rev Méd Chile 2005; 133: 403-8.

19. Quiñones-Baldrich W, Panetta T, Vescera C, KashYAP V. Repair of type IV thoracoabdominal aneurysm with a combined endovascular and surgical approach. J Vasc Surg 1999; 30: 555-60.

20. RuBIN B. Extra-anatomic Visceral Revascularization and Endovascular Stent-Grafting for Complex Thoracoabdominal Aortic Lesions. Pers Vasc Surg Endovasc Ther 2005; 17: 227-36.

21. ReiLy J, Rubin B, Thompson R, Auen B, Anderson C, SICARD G. Long-term effectiveness of extraanatomic renal artery revascularization. Surgery 1994; 116: 784-90.

22. Johnston K, LndSAY T, WALKer P, Kalman P. Mesenteric arterial bypass grafts: Early and late results and suggested surgical approach for chronic and acute mesenteric ischemia. Surgery 1995; 118: 1-7.

23. McMilan W, McCarthy W, Bresticker M, Pearce W, Schneider J, Golan J ET aL. Mesenteric artery bypass: objective patency determination. J Vasc Surg 1995; 21: 729-41.

24. Jacobs T, Won J, Gravereaux E, Faries P, Morrisey $\mathrm{N}$, Teodorescu V et al. Mechanical failure of prosthetic human implants: A 10-year experience with aortic stent graft devices. J Vasc Surg 2003; 37: 16-26.

25. Clouse W, Marone L, Davison J, Dorer D, Brewster D, LAMuRAGLA G ET AL. Late aortic and graft-related events after thoracoabdominal aneurysm repair. J Vasc Surg 2003; 37: 254-61.

26. Greenberg R, Haulon S, Lyden S, Srivastava S, Turc A, EAgleton M ET al. Endovascular management of juxtarenal aneurysms with fenestrated endovascular grafting. J Vasc Surg 2004; 39: 279-87.

27. CHUTER T. Branched and fenestrated stent grafts for endovascular repair of thoracic aortic aneurysms. J Vasc Surg 2006; 43 Suppl A: 111A-115A.

28. Haddad F, Greenberg R, Walter E, Naluy J, O’NeiL S, KoLN G ET AL. Fenestrated endovascular grafting: The renal side of the story. J Vasc Surg 2005; 41: 181-90. 